Z Gerontol Geriat 2011 · 44:145-145

DOI 10.1007/s00391-011-0188-z

Online publiziert: 15. Mai 2011

(c) Springer-Verlag 2011

\author{
M. Gogol ${ }^{1} \cdot R$. Thiesemann ${ }^{2}$ \\ ${ }^{1}$ Klinik für Geriatrie, Krankenhaus Lindenbrunn, Coppenbrügge \\ ${ }^{2}$ Klinik für Geriatrie, Fabricius-Klinik Remscheid GmbH
}

\title{
Kardiovaskuläres Altern
}

Kardiovaskuläre Erkrankungen spielen, auch bei erst subklinischer Manifestation, eine bedeutende Rolle für den Alterungsprozess des Organismus, das Auftreten funktioneller Einschränkungen und frühzeitiger Mortalität. Aus Anlass des 70. Geburtstags von Christoph Lucke, dem während seiner ganzen Berufszeit amputierte Alterspatienten ein besonderes Anliegen in der Klinik und in der Forschung waren, wurde beim Kongress der Deutschen Gesellschaft für Geriatrie 2009 in Göttingen zum Thema kardiovaskuläres Altern ein Symposium veranstaltet. Geriatrische Syndrome wie Frailty [1] und andere sind mit dem Auftreten von kardiovaskulären Erkrankungen assoziiert $[2,3]$.

Zum Thema oxidativer Stress und Proteinglykierung durch ,advanced glycation endproducts" (AGE) beschreibt A. Simm, Halle, die Bedeutung dieses noch recht jungen Biomarkers, der über kardiometabolische Erkrankungen hinaus [4] auch eine Rolle bei anderen Entitäten, z. B. bei der chronisch-obstruktiven Lungenerkrankung (COPD [5, 6]) spielt.

"Inflammageing“ ist ein Thema, dem sich D. Schmidt, Coppenbrügge, widmet und die derzeit meistdiskutierten Biomarker am Beispiel kardiovaskulärer Erkrankungen diskutiert und in einen Kontext mit anderen Erklärungsmodellen des Alterns stellt. Wichtigste und nicht überraschende Feststellung dieses sich stürmisch entwickelnden Forschungsgebiets [7] ist, dass es den einen und/oder umfassenden Biomarker nicht gibt.

Die chronische Herzinsuffizienz ist eine der häufigsten und schwerwiegendsten Manifestationsformen kardialer Erkrankungen und zählt zu den Top-5-Diagnosen für funktionale Einschränkungen, reduzierte Lebensqualität und Mortalität. $D$. Fischer, Hannover, diskutiert die allgemei- nen Empfehlungen zur Therapie der Herzinsuffizienz, Neuerungen im Rahmen der gering invasiven Klappenrekonstruktion sowie der Koronarrevaskularisierung. Problematisch ist und bleibt der Umstand, dass die klinische Forschung bei Alterspatienten bisher wenig positive Ergebnisse für die Behandlung der Herzinsuffizienz erbracht hat. Ein häufiger Grund ist der Ausschluss hochaltriger Menschen in den klinischen Studien $[8,9]$ - ein Umstand, der sich über die Jahre leider nicht verbessert hat, wie eine jüngst publizierte Studie am Beispiel des akuten Koronarsyndroms zeigt [10].

Die Besonderheiten der Diagnostik des Myokardinfarktes beim älteren Menschen stellt $P$. Bahrmann, Nürnberg, dar. Anhand eigener Forschungsergebnisse zeigt er Hinweise dafür, dass die Sensitivität von laborchemischen Ergebnissen einer sorgfältigen Analyse bedarf, da beim älteren Menschen schon die krankheitsfreien Basalwerte höher liegen als beim jüngeren Patienten.

Den Zusammenhang zwischen metabolischen und kardiovaskulären Erkrankungen beschreibt A. Bahrmann, Heidelberg. Trotz dieses Zusammenhangs zeigt die Arbeit, dass die Zielsetzung einer antidiabetischen Therapie beim älteren Menschen individuell erfolgen muss und ein enger Zusammenhang mit dem Ausmaß des glykolysierten Hämoglobins nicht besteht.

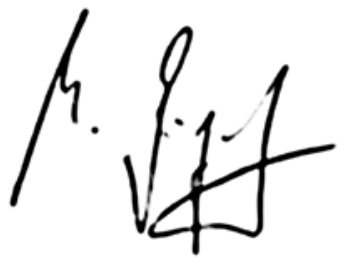

M. Gogol

\section{Korrespondenzadresse \\ Dr. M. Gogol}

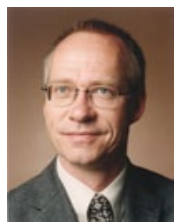

Klinik für Geriatrie,

Krankenhaus Lindenbrunn

Lindenbrunn 1,

31863 Coppenbrügge gogol@krankenhaus-

lindenbrunn.de

\section{Literatur}

1. Afilalo J, Karunananthan S, Eisenberg MJ, Alexander KP, Bergman H (2009) Role of frailty in patients with cardiovascular disease. Am J Cardiol 103:1616-1621

2. Huerre C, Guiot A, Maréchaux S et al (2010) Functional decline in elderly patients presenting with acute coronary syndromes: impact on midterm outcome. Arch Cardiovasc Dis 103:19-25

3. Rosso AL, Eaton CB, Wallace R et al (2011) Combined impact of geriatric syndromes and cardiometabolic diseases on measures of functional impairment. J Gerontol 66A:349-354

4. Semba RD, Nicklett EJ, Ferrucci L (2010) Does accumulation of advanced glycation end products contribute to the aging phenotype? J Gerontol 65:963-975

5. Wu L, Ma L, Nicholson LF, Black PN (2011) Advanced glycation end products and its receptor (RA$\mathrm{GE})$ are increased in patients with COPD. Respir Med 105:329-336

6. Smith DJ, Yerkovich ST, Towers MA et al (2011) Reduced soluble receptor for advanced glycation end-products in COPD. Eur Respir J 37:516-522

7. Müller-Werdan U (2007) Inflammation and ageing. Z Gerontol Geriatr 40:362-365

8. Heiat A, Gross CP, Krumholz HM (2002) Representation of the elderly, women, and minorities in heart failure clinical trials. Arch Intern Med 162:16821688

9. Kitzman DW, Rich MW (2010) Age disparities in heart failure research. JAMA 304:1950-1951

10. Dodd KS, Saczynski JS, Zhao Y, Goldberg RJ, Gurwitz JH (2011) Exclusion of older adults and women from recent trials of acute coronary syndromes. J Am Geriatr Soc 59:506-511 